\title{
A importância do exame cito-anuscópico sob visão ampliada para 0 diagnóstico das neoplasias intra-epiteliais anais em pacientes com neoplasias intra-epiteliais genitais
}

The role of cytology and high-resolution anoscopy in the diagnosis of anal intraepithelial neoplasia in women with genital intraepithelial neoplasia

\author{
Cláudia Jacyntho ${ }^{1}$, Paulo Giraldo ${ }^{2}$
}

Inspirados nos trabalhos de Palefsky ${ }^{1}$, pioneiro no estudo da investigação de lesões precursoras do câncer de ânus, sobretudo em homens homo e bissexuais HIV positivos, propusemo-nos a avaliar a prevalência de neoplasia intra-epitelial anal (NIA) em mulheres com neoplasias intraepiteliais genitais (NIG), compreendendo as cervicais - NIC, as vaginais - NIVA, as vulvares NIV e as perianais - NIPA.

Recrutaram-se pacientes do ambulatório de Colposcopia do Hospital dos Servidores do Estado (HSE-RJ-MS) e do Ambulatório de Infecções Genitais do Departamento de Tocoginecologia da Unicamp, com diagnóstico histopatológico de NIG, submetendo-as ao estudo cito-anuscópico sob visão ampliada, após assinatura do termo de consentimento livre e esclarecido. Todos os casos que apresentaram lesões anais suspeitas de NIA foram biopsiados com pinça de Medina e o material foi enviado para estudo histopatológico para o serviço de anatomia patológica do HSE- RJ-MS.

Os resultados preliminares acenam para achados altamente intrigantes. Entre os primeiros 100 casos investigados encontraram-se $12 \%$ de NIA (NIA de alto grau - 1 caso e NIA de baixo grau - 11 casos) confirmados com estudo histológico e $18 \%$ de alterações citológicas (lesões intra-epiteliais de baixo grau - 3 casos e ASCUS 15 casos). A anuscopia ajudou na identificação dos casos a serem biopsiados tendo identificado achados anormais (maiores ou menores) em 19\% das mulheres investigadas. O caso de NIA de alto grau foi identificado em paciente portadora de NIC e NIV de alto grau concomitantes. Dos 11 casos de NIA de baixo grau, cinco tinham NIC de baixo grau isolado, dois tinham, além do NIC de baixo grau, outra neoplasia intra-epitelial associada. Dois casos apresentaram doença multicêntrica completa (NIC, NIV, NIVA e NIPA) e dois casos não tiveram qualquer acometimento do colo uterino (NIVA/NIPA).

Nas últimas publicações de Palefsky ${ }^{1}$ e Goldie et $a .^{2}$ eles referem que outros grupos, além dos homens homo e bissexuais HIV positivos, podem beneficiar-se do rastreamento anal, dentre eles, as mulheres com lesões de alto grau cervicais, vaginais ou vulvares, objeto deste estudo.

Hernandez e Goodman ${ }^{3}$ demonstraram, em estudo preliminar, que mulheres com infecção por HPV no colo uterino apresentam risco duas vezes maior para a presença de infecção por HPV anal concomitante. Alguns anos antes, Moscicki et al. ${ }^{4}$ referiram que mulheres jovens praticantes de sexo anal ou com história positiva para NIC apresentaram risco maior para citologias anais anormais.

Provavelmente os mesmos fatores que levaram à persistência da NIG influenciaram no aparecimento da NIA. Os dados agora apresentados, embora de caráter preliminar, são altamente expressivos, uma vez que a maioria de pacientes recrutadas neste estudo são HIV negativas e imunologicamente competentes, sugerindo que a prevenção de câncer de ânus em mulheres com NIG pode ser uma necessidade em nosso meio. Poucos estudos vêm sendo realizados em mulheres e acreditamos que este seja o primeiro a triar a NIA em mulheres com NIC e/ou NIVA e/ou NIV e/ou NIPA no Brasil.

Faz-se necessário agora aumentar a casuística de mulheres investigadas, bem como incluir no estudo um grupo-controle de mulheres que não tenham NIG.

Este trabalho foi aprovado pelos Comitês de Ética e Pesquisa das instituições envolvidas

\footnotetext{
1 Setor de Colposcopia do Hospital dos Servidores do Estado do Rio de Janeiro - Ministério da Saúde.

2 Ambulatório de Infecções Genitais do Centro de Atenção Integral à Saúde da Mulher (CAISM) - UNICAMP - São Paulo.

Correspondência: Cláudia Jacyntho

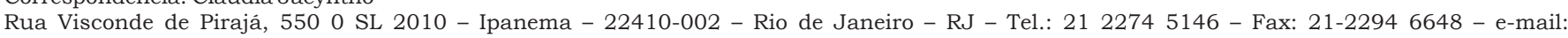
jacyntho@jacyntho.com.br

Recebido em: 30/11/2004 Aceito com modificações em: 20/12/2004
} 
PALAVRAS-CHAVE: HPV. Neoplasia intraepitelial anal. Colo: lesões pré-neoplásicas. Câncer: ânus.

KEYWORDS: HPV. Anal intraepithelial neoplasia. Cervical intraepithelial neoplasia. Anal cancer prevention

\section{Referências}

1. Palefsky JM. Anal and perianal lesions AIN in HIV+ and HIV- women. Annals of the $21^{\text {st }}$ International Conference \& Clinical Workshop Papillomavirus; 2004 Feb 20-26; Mexico City, Mexico. Mexico
City: International Papillomavirus Society; 2004. p. 25-6.

2. Goldie S, Palefsky JM, Workowsi K. Anal cancer in HIV infection: to screen or not to screen? AIDS Clin Care. 2004; 16:53-5, 57.

3. Hernandez BY, Goodman MT. Concurrent cervical and anal HPV infection among a multiethnic cohort of women in Hawaii. Annals of the $21^{\text {st }}$ International Conference \& Clinical Workshop Papillomavirus; 2004 Feb 20-26; Mexico City, Mexico. Mexico City: International Papillomavirus Society; 2004. p. 115.

4. Moscicki AB, Hills NK, Shiboski S, et al. Risk factors for abnormal anal cytology in young heterosexual women. Cancer Epidemiol Biomarkers Prev. 1999; 8:173-8. 